\title{
Inserção das ciências básicas no currículo integrado do curso de Medicina da Universidade Estadual de Londrina
}

\author{
The participation of basic sciences in the \\ integrated curriculum of the Londrina State \\ University medical school
}

Lúcia Helena Mendonça Vargas ${ }^{1}$ Ilce Mara de Syllos Colus ${ }^{1}$ Rosa Elisa Carvalho Linhares ${ }^{1}$ Tereza Maria Sandis Salomão ${ }^{1}$ Maurício de Castro Marchese ${ }^{1}$

\section{PALAVRAS-CHAVE}

- Ciências Básicas;

- Currículo Integrado;

- Aprendizagem Baseada em Problemas.
Recebido em: 29/06/2007 Reencaminhado em: 11/10/2007 Reencaminhado em: 05/12/2007 Aprovado em: 20/01/2008
REVISTA BRASILEIRA DE EDUCAÇ̃̃O MÉDICA $174 \frac{\text { 22 (2) : } 174-179: 2008}{32}$

\footnotetext{
${ }^{1}$ Universidade Estadual de Londrina, Paraná, Brasil.
}

\begin{abstract}
A B S T R A C T
The medical school of the State University of Londrina established an integrated curriculum using Problem Based Learning (PBL) as teaching strategy in the thematic modules of the medical course. Although according to the literature Problem Based Learning enhances the understanding and retention of basic sciences concepts, some authors fear that these basic concepts are not sufficiently emphasized in this methodology. The objective of this study is to evaluate the participation of basic sciences in the integrated curriculum of the Londrina State University medical school. To this purpose a semi-structured questionnaire was elaborated for evaluating the participation of teachers of the basic areas in the different activities making part of the current curriculum. There was an increase of $36 \%$ in the participation of basic science teachers in relation to the previous curriculum. It was verified that basic sciences teachers participated in activities of the integrated curriculum during the first four but mainly during the first two years. Efforts should be made for achieving a more intense integration of basic sciences and clinical practice throughout the course.
\end{abstract}




\section{INTRODUÇÃO}

Na década de 1920, as mudanças propostas por Flexner e seus colegas para as escolas médicas dos Estados Unidos resultaram em crescimento, fortalecimento e independência dos departamentos de ciências básicas. Tais ocorrências, somadas ao surgimento de inúmeras especialidades clínicas, contribuíram para a fragmentação e duplicação dos currículos ${ }^{1}$. Desta forma, os currículos vêm sofrendo a pressão da explosão do conhecimento científico e de seus meios de divulgação, que resulta em sobrecarga do cognitivo e pulverização dos conhecimentos.

Historicamente, os currículos têm seu modelo organizado em disciplinas isoladas, desvinculadas da realidade profissional, hipertrofiadas em conteúdos, voltadas para uma formação tecnicista e para especialidades, não formando profissionais que atendam às necessidades de saúde da sociedade. Neste contexto, as disciplinas das ciências básicas, de cunho científico e estrutural, constituem um alicerce para a composição do entendimento do ser humano como conjunto composto por um sistema biológico e uma estrutura psicológica e social. Conforme Wannmacher ${ }^{2}$, os professores das disciplinas básicas se empenham em adequar uma visão integrada de todas as disciplinas e "convencer os alunos" de que os conteúdos de suas disciplinas terão certamente uma aplicação prática em algum momento da carreira acadêmica e profissional.

Nos últimos anos, tem se produzido intenso debate em torno da reforma curricular das escolas médicas no Brasil ${ }^{3,4}$. Um dos maiores desafios para o sucesso dessas mudanças é a adoção de uma visão mais holística e de métodos mais participativos. De acordo com Batista ${ }^{5}$, construir caminhos no ensino médico a partir da interdisciplinaridade inclui a implementação de desenhos curriculares que possibilitem a articulação de conteúdos, valorizem o enfoque "problematizador" e desenvolvam atividades acadêmicas que tenham como eixos a prática médica no contexto do trabalho em saúde e a inserção do estudante e do professor como sujeitos na produção contextualizada de saberes.

O curso de Medicina da Universidade Estadual de Londrina (UEL) foi uma das escolas que se propôs a enfrentar esse desafio. Desde 1998, iniciou um processo de implantação e desenvolvimento de um currículo médico integrado ${ }^{6}$. Nas quatro primeiras séries, a estrutura do currículo é formada por módulos temáticos interdisciplinares, módulos de habilidades clínicas e práticas de ensino serviço e comunidade, e nos dois últimos anos ocorre o internato médico. Nos módulos temáticos, um conjunto de temas correlatos com conteúdos das ciências básicas e clínicas é desenvolvido de forma integrada com problemas prioritários de saúde da população. Nesses módulos temáticos, a metodologia utilizada é a Aprendizagem Baseada em Problemas (ABP). Ape- sar de dados da literatura apontarem que o uso da metodologia ABP aumenta o entendimento e a retenção de conceitos das ciências básicas ${ }^{7,8}$, alguns autores demonstram preocupação com o fato de as ciências básicas não receberem suficiente ênfase na metodologia $\mathrm{ABP}^{1,9,10}$. Entretanto, o medo exagerado dos docentes em não cobrir todo o conteúdo das antigas disciplinas tem sido apontado como um dos fatores de desgaste do conjunto de princípios interligados que fazem a $\mathrm{ABP}$ funcionar ${ }^{11}$.

Considerando os fatos relatados, este trabalho objetivou avaliar a inserção das ciências básicas no currículo integrado do curso de Medicina da UEL, quantificando e comparando a participação dos docentes antes e depois da inserção do currículo integrado.

\section{METODOLOGIA}

Foi elaborado um questionário semi-estruturado com sete questões fechadas (Anexo) sobre o processo de inclusão docente nas várias atividades desenvolvidas no atual currículo.

A participação dos docentes foi verificada por meio da aplicação de questionário semi-estruturado ao universo dos docentes das áreas das ciências básicas que atuam ou atuaram no curso de Medicina da UEL. As questões de 1 a 5 foram respondidas pelos coordenadores de área e/ou chefes de departamentos, e as questões 6 e 7 pelos docentes envolvidos no curso. As áreas incluídas como ciências básicas foram: Anatomia, Biofísica, Biologia Celular, Bioquímica, Embriologia, Farmacologia, Fisiologia, Genética, Histologia, Imunologia, Microbiologia, Parasitologia e Patologia Geral. Os dados foram tabulados, e as porcentagens foram calculadas. Todos os docentes e administradores que responderam o questionário foram esclarecidos sobre os objetivos do trabalho, e houve consentimento verbal por parte deles.

Para se ter uma visão de processo, foram coletados dados sobre a participação dos docentes das ciências básicas no final da implantação do currículo integrado nas quatro primeiras séries, por meio de consultas aos manuais dos módulos temáticos do ano de 2002. Estes foram tabulados considerando-se o número de docentes do básico nas diferentes funções do curso. Foram consideradas como atividades-fim (acadêmicas) as seguintes funções: professor-tutor, instrutor de atividades práticas e palestrante. A participação nos módulos temáticos interdisciplinares em atividades acadêmicas está bem estabelecida na literatura ${ }^{12,14}$ e no projeto político-pedagógico do curso de Medicina da UEL $^{15,16}$ e está descrita a seguir:

- Professor tutor - atua em pequenos grupos como facilitador/mediador, na construção do conhecimento pelos estudantes, por meio da resolução de problemas.

- Professor instrutor de atividades práticas - orienta uma aula prática sobre um tema específico do módulo temático; 
- Professor palestrante-ministra aulas teóricas expositivas sobre um tema específico do módulo temático.

Também se verificou a participação dos docentes em atividades-meio: coordenação, vice-coordenação e membros de planejamento de módulos; coordenação de série e participação nas comissões de apoio administrativo didático-pedagógico ao Colegiado de Curso de Medicina: Comissão de Apoio Discente e Docente (CADD), Comissão de Avaliação (CAV), Comissão de Acompanhamento Curricular (CAC) e Comissão de Educação Permanente Docente (CEPD).

Para facilitar a leitura dos dados, as tabelas foram ajustadas e simplificadas.

\section{RESULTADOS E DISCUSSÃO}

Ao compararmos o número de docentes das ciências básicas que atuavam antes da mudança do currículo para o modelo atual, observamos um aumento de 39 docentes para 53 (36\%). A distribuição do número de docentes das diferentes áreas de conhecimento, antes e depois da reforma curricular, está apresentada na Tabela 1. Este fato indica que o currículo integrado necessita de envolvimento de maior número de docentes, uma vez que as áreas básicas estão inseridas nas quatro primeiras séries do curso, nas quais as atividades, muitas vezes, são concomitantes. Para o início das atividades do novo currículo do curso de Medicina da UEL, foi preciso capacitar os docentes que atuariam como professores-tutores e coordenadores de módulos. Houve uma destacada participação de docentes da área básica nos cursos de capacitação realizados ${ }^{17}$. Até o momento, 58 docentes da área básica foram capacitados. Atualmente, 37 docentes da área básica exercem efetivamente o papel de professores-tutores nos diversos módulos temáticos das quatro primeiras séries.

\section{TABELA 1}

Distribuição do número de docentes das diferentes áreas básicas de conhecimento antes e depois da reforma curricular

\begin{tabular}{lcc}
\hline & \multicolumn{2}{c}{ Número de docentes } \\
\cline { 2 - 3 } Áreas de conhecimento & Antes & Após \\
\hline Anatomia & 4 & 3 \\
Biofísica & 1 & 1 \\
Biologia Celular & 1 & 2 \\
Bioquímica & 2 & 5 \\
Embriologia & 1 & 4 \\
Farmacologia & 6 & 3 \\
Fisiologia & 6 & 7 \\
Genética & 1 & 3 \\
Histologia & 4 & 8 \\
Imunologia & 2 & 5 \\
Microbiologia & 2 & 5 \\
Parasitologia & 5 & 3 \\
Patologia Geral & 4 & 5 \\
\hline
\end{tabular}


A atuação dos docentes das ciências básicas como professores-tutores está distribuída nas quatro séries, na seguinte proporção: $52 \%(n=32)$ na primeira série; $35 \%(n=21)$ na segunda; $10 \%$ $(n=6)$ na terceira; e 3\% $(n=2)$ na quarta série. A Figura 1 mostra que a participação dos professores-tutores das ciências básicas aumentou durante o processo de implantação do currículo integrado, considerando o ano de 2002 e atualmente (ano de 2006).

Nas atividades práticas realizadas nos laboratórios das diferentes áreas básicas, a participação dos professores-instrutores está distribuída da seguinte maneira: $50 \%(n=29)$ na primeira série; $25 \%(n=15)$ na segunda; $20 \%(n=12)$ na terceira; e $5 \%(n=$ 3) na quarta série. Ao contrário do ocorrido com a participação dos docentes das ciências básicas como professor-tutor, pode-se observar que houve uma diminuição da participação docente nas atividades práticas das duas primeiras séries, sendo esta redução de $25 \%$ na primeira série e de $52 \%$ na segunda. Mas houve aumento na terceira série (30\%) e manutenção na quarta série (Figura 1).

As palestras proferidas por docentes das ciências básicas compõem $43 \%(n=16), 38 \%(n=14), 13,5 \%(n=5)$ e $5,5 \%(n=2)$ na primeira, segunda, terceira e quarta séries, respectivamente. Com exceção da primeira série, em que ocorreu uma diminuição de $20 \%$, o número de palestrantes das ciências básicas em 2002 não diferiu significativamente do atual (Figura 1).

\section{FIGURA 1}

Número de docentes das Ciências Básicas envolvidos nas atividades acadêmicas-fim em relação aos anos de 2002 e 2006.

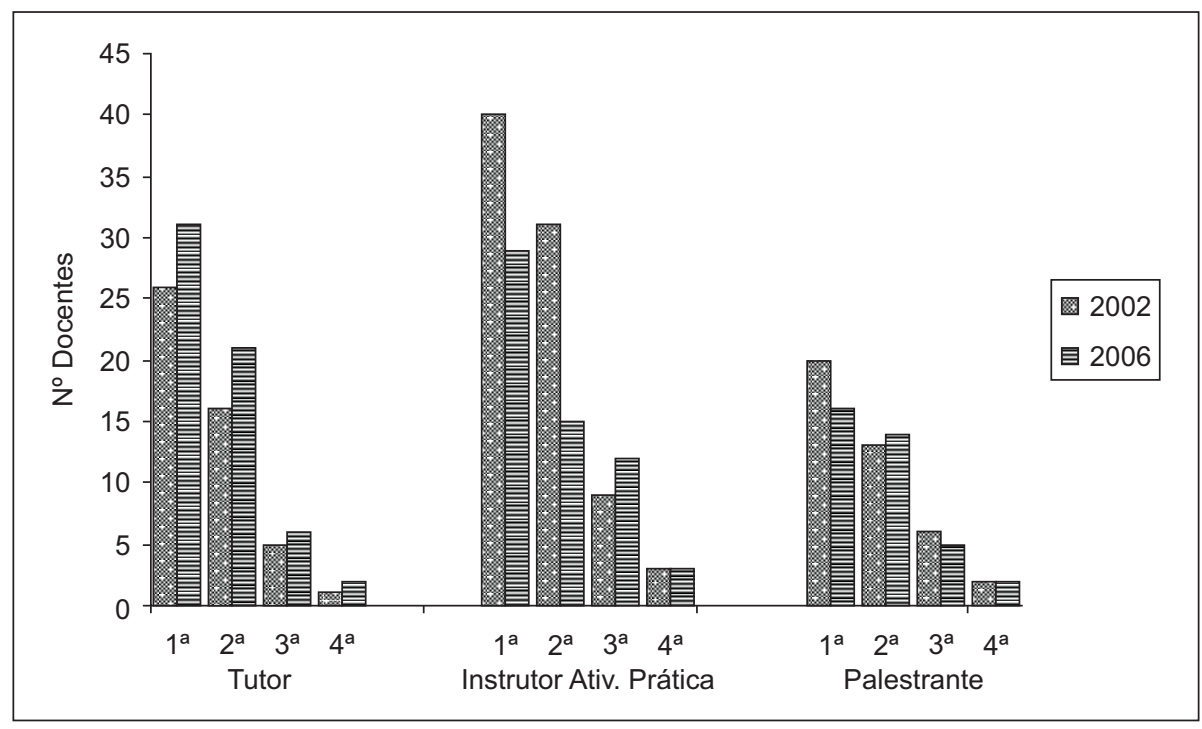

Das 13 áreas consultadas no presente estudo, seis consideraram satisfatória a inserção de seus conteúdos programáticos no atual currículo; sete avaliaram como pouco satisfatória sua inserção; e nenhuma área considerou excelente ou insuficiente sua participação. Apesar de dados da literatura apontarem que o uso da metodologia ABP aumenta o entendimento e a retenção de conceitos das ciências básicas ${ }^{13}$, alguns autores demonstram preocupação com o fato de as ciências básicas não receberem suficiente ênfase nesta metodologia. $\mathrm{O}^{\prime} \mathrm{Neill}^{8}$ relata que, na metodologia de $\mathrm{ABP}$, problemas com aspectos clínicos relevantes são descritos como estímulo para discussão e veículo da aprendizagem das ciências básicas. Uma desvantagem é que os estudantes podem se tornar mais interessados nos aspectos clínicos do problema e negligenciar o conhecimento das ciências básicas. Outro aspecto é que os estudantes não adquirem estrutura apropriada para a contínua aprendizagem das ciências básicas ${ }^{8}$. Porém, o medo exagerado dos docentes em não cobrir todo o conteúdo das antigas disciplinas tem sido apontado como um dos fatores de desgaste do conjunto de princípios interligados que fazem a ABP funcionar ${ }^{11}$.

A participação das ciências básicas nas atividades-meio, tais como coordenação de série e de módulo temático, vem ocorrendo desde a implantação do currículo integrado, exclusivamente na primeira e na segunda séries. Entretanto, a participa- 
ção dos docentes das ciências básicas como membros de planejamento de módulo e vice-coordenadores de módulo está distribuída nas quatro séries, embora com predominância nas duas primeiras. A diminuição observada entre os períodos de
2002 e o atual no número de docentes como membros de grupo de planejamento de módulo é justificada pelo processo de construção inicial dos módulos, que demandava grande participação docente (Figura 2).

\section{FIGURA 2}

Número de docentes das Ciências Básicas envolvidos nas atividades de estruturação e organização dos módulos temáticos em relação aos anos de 2002 e 2006.

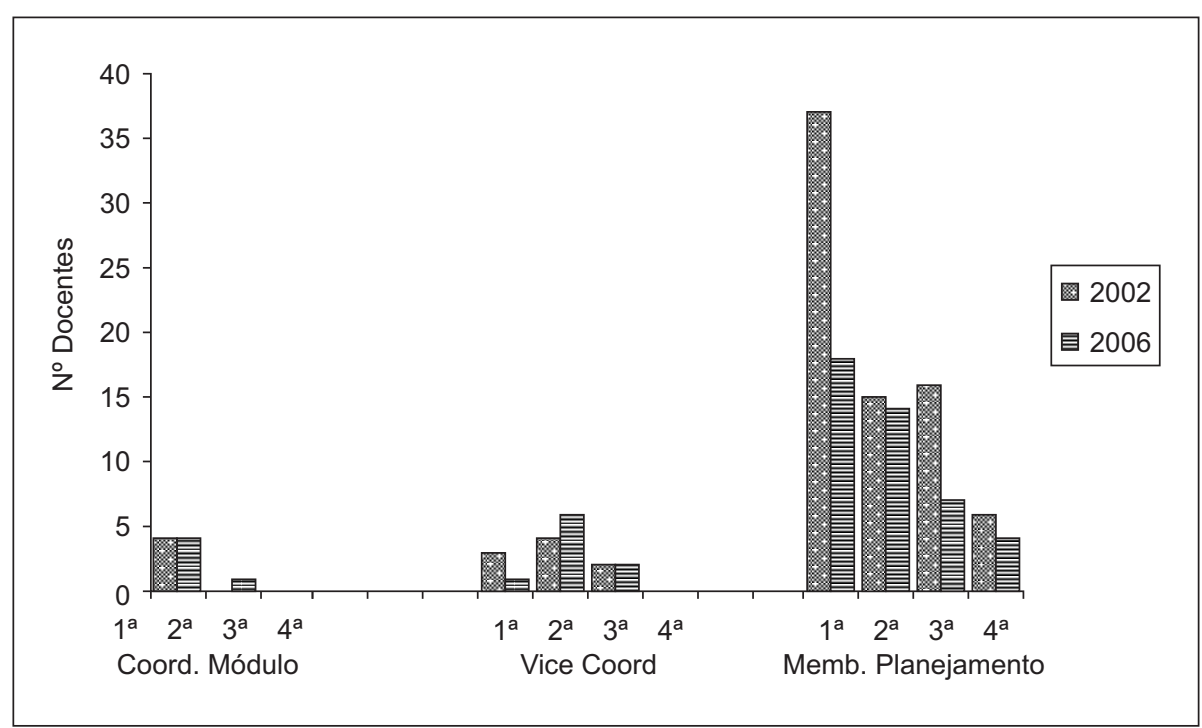

OColegiado do curso de Medicina conta com o apoio de diferentes comissões, compostas por docentes das áreas básicas e clínicas que sempre tiveram a participação de docentes das áreas básicas, atuando, inclusive, como coordenadores. Atualmente, três das quatro comissões são coordenadas por docentes das áreas básicas: CADD, CAC e CEPD.

Pode-se concluir que a implantação do currículo possibilitou maior participação docente nas diversas atividades ao longo das quatro séries, com ênfase nas duas primeiras. Quanto à inserção dos conteúdos das ciências básicas, observou-se que aproximadamente metade das áreas a considerou pouco satisfatória, enquanto as demais avaliaram sua inserção como satisfatória. Devem ser feitos esforços para que a inserção das ciências básicas possa ocorrer com maior intensidade em todas as séries.

A Comissão de Acompanhamento Curricular está realizando estudos na tentativa de analisar qualitativamente a inserção e integração dos conteúdos das ciências básicas na formação geral do médico.

\section{REFERÊNCIAS}

1. Sweeney G. The challenge for basic science education in problem-based medical curricula. Clinical and Investigate Medicine 1999; 22(1): 15-22. [Online]. Disponível em: http:/ / www.cma.ca [Acesso em: 26 de julho de 2000].

2. Wannmacher CMD. Ensinando Bioquímica para futuros médicos. Rev. Bras. Ens. Bioq. Biol. Molec.[periodico na Internet]. 2001;1:12. Disponível em: http:/ / www.sbbq.org.br/revista/artigo.php?artigoid=1. [Acesso em: 26 de julho de 2000]

3. Almeida MJ. Educação Médica e Saúde: possibilidades de mudanças. Londrina/Rio de Janeiro: EDUEL/Associação Brasileira de Educação Médica; 1999.

4. Rede Unida. A construção de modelos inovadores de ensino- aprendizagem:as lições aprendidas pela rede UNIDA. Divulgação em Saúde Debate 2000; 22:49-62.

5. Batista SHS. A interdisciplinaridade no ensino médico. Rev. Bras. Educ. Med. 2006; 30 (1): 39-46. 
6. Campos JJB. Como abrir caminhos para a transformação do ensino médico no Brasil. Rev. Bras. Educ. Méd. 1999; 23 (2/3):11-20.

7. Dods RF. An action research study of the effectiveness of problem-based learning in promoting the acquisition and retention of knowledge. J. Educ. Gifted. 1997; 20: 423-437.

8. Finucane PM, Johnson SM, Prideaux DJ. Problem basic learning; its rationale and efficacy. Med. J. Aust. 1998; 168: 445-448.

9. Anderson WL, Glew RH. Support of problem-based learning curriculum by basic science faculty. Med. Education 2002; 7:10 [Online].Disponível em: http//www.med-ed-online.org [Acesso em: 26 de maio de 2007].

10. O'neill PA. The role of basic sciences in a problem-based learning clinical curriculum. Med. Education 2000; 30: 608-613.

11. Moust JHC, van Berkel HJM, Schmidt HG. Sign of erosion: Reflections on the three decades of problem-based learning at Maastricht University. Higher Education. 2005; 50: 665-683.

12. Venturelli J, Funciones Y. Características de Los Docentes: funcionamiento en um programa centrado en el estudiante y basado en la solución de problemas. In: Venturelli, J. Educacion Médica: nuevos enfoques, metas y métodos. Washington: Publicación de la Organización Panamericana de la Salud/ Oficina Regional de la Organización Mundial de la Salud; 1997. [Série PALTEX Salud y Sociedad n.5, 2000]

13. Schimidt HG. Foundations of Problem-Based Learning: Some Explanatory Notes. Med. Education 1993; 27: 422-432.
14. Wilkerson LA, Hundert EM. Becoming a Problem-based Tutor: Increasing Self-awereness Throgh Facility Development. In: Boud D, Feletti GI. (editores) 2.ed. London: Kogan Page Limited; 2001. p. 160-171. G.I.

15. Universidade Estadual de Londrina, Centro de Ciências da Saúde, Colegiado do Curso de Medicina. Projeto Político Pedagógico do Curso de Medicina (PPPMed). Versão V. Londrina: Colegiado do Curso de Medicina/Centro de Ciências da Saúde; 2004.

16. Universidade Estadual de Londrina. Centro de Ciências da Saúde. Colegiado do Curso de Medicina. Atos Legais de Consolidação do Currículo Integrado. Londrina: Colegiado do Curso de Medicina/ Centro de Ciências da Saúde/UEL; 2006.

17. Lima GZ, Almeida HGG, Ferreira Filho OF, Linhares REC, Oberdiek HI, Colus IMS. Apredizagem baseada em Problemas (ABP): Construindo a Capacitação em Londrina. Rev. Bras. Educ. Méd. 2003; 27: 5-11.

\section{CONFLITOS DE INTERESSE:}

Declarou não haver

\section{ENDEREÇO PARA CORRESPONDÊNCIA}

Lúcia Helena Mendonça Vargas

Centro de Ciências Exatas

Departamento de Bioquímica e Biotecnologia

Rodovia Celso Garcia Cid (PR 445), km 380

Cep: 86051-990

Cx Postal 6001

Londrina - Paraná - Brasil

luciavargas@uel.br 